PsyArxiv preprint first posted online March 3, 2017, http://osf.io/vaqk3/. The copyright holders for this preprint (which was not peer-reviewed) are the authors. It is made available under a CC-BY-NC-ND 4.0 International license.

Word order patterns in gesture are sensitive to modality-specific production constraints

\title{
Melissa Kline
}

Miguel Salinas

Eunice Lim

Evelina Fedorenko

Edward Gibson 
PsyArxiv preprint first posted online March 3, 2017, http://osf.io/vaqk3/. The copyright holders for this preprint (which was not peer-reviewed) are the authors. It is made available under a CC-BY-NC-ND 4.0 International license.

\begin{abstract}
A fundamental typological variation in the world's languages is their basic word order; around $80 \%$ of spoken languages are either Subject-Object-Verb (SOV) or Subject-Verb-Object (SVO). Previous work has related this typological pattern to a striking finding in ad-hoc gesture production: across a wide range of languages, people tend to use the order SOV to gesture events with inanimate patients, and SVO orders for those with animate patients (Gibson, Piantadosi, et al., 2013; Hall, Mayberry, \& Ferreira, 2013, i.a.). Gibson et al. (2013) interpret this evidence as support for a noisy channel model of communication, under which producers attempt to reduce ambiguity for comprehenders. On the other hand, this pattern might also result from the particular kinds of gestures that people tend to use for different kinds of events (Hall et al. 2013). We conducted two production studies - one in gesture, one using non-iconic written symbolsdesigned to modify the communication task in different ways. Two main findings emerged from these tests: first, simple modifications to the gesture paradigm can have a profound effect on the orders used: the instructions given in Experiment 1 (gesture) dramatically affected the use of SVO orders, even though participants were gesturing about the same events under the same communicative contexts. Second, there is an apparent association between the physical form of the gestures used to communicate and word order: we found that participants were most likely to use SVO orders in Experiment 1 for animate-animate events when using body-based gestures (avoiding role conflict, as described by Hall et al. 2013); furthermore in Experiment 2 (written symbols), there was no association between SVO ordering and animate-animate scenes (contrary to predictions of the noisy channel model). We conclude that gesturing paradigms, while a striking and naturalistic example of ad-hoc communication dynamics, are also sensitive to the
\end{abstract}


PsyArxiv preprint first posted online March 3, 2017, http://osf.io/vaqk3/. The copyright holders for this preprint (which was not peer-reviewed) are the authors. It is made available under a CC-BY-NC-ND 4.0 International license.

particular modality and task instructions, and as such do not provide straightforward evidence for noisy channel theories of word order typology.

Abstract word count: 320 
PsyArxiv preprint first posted online March 3, 2017, http://osf.io/vaqk3/. The copyright holders for this preprint (which was not peer-reviewed) are the authors. It is made available under a CC-BY-NC-ND 4.0 International license.

\section{Introduction}

A fundamental typological variation in human language is basic word order: in English, a sentence like The boy kicks the ball expresses the agent, action and patient in that order (or subject, verb, and object, SVO) ${ }^{1}$ of an event. In Korean, the subject and object are typically expressed before the verb (SOV):

$$
\begin{aligned}
& \text { 소년이 공을 찬다 } \\
& \begin{array}{llllll}
\text { sonyen } & -\mathrm{i} & \mathrm{k} \text { kng } & \text {-ul } & & \\
& & & & & \\
\text { boy } & \text {-NOM } & \text { ball } & \text {-ACC } & \text { kick } & \text {-DEF }
\end{array}
\end{aligned}
$$

\section{The boy kicks the ball}

Strikingly, though there are six possible permutations (SVO, SOV, OSV, OVS, VSO, VOS), most languages are either SVO like English (41\%) or SOV like Korean (47\%) the most common pattern is languages with free word order (i.e. no fixed preference), followed by the other four basic permutations (Dryer, 2005).

Evidence from signed languages, patterns of historical language change, and gesturecommunication experiments suggests that one word order, SOV, might be somehow primary in cognition and language (Gell-Mann \& Ruhlen, 2011; Givón, 1979; Goldin-Meadow \& Feldman, 1977; Goldin-Meadow, So, Özyürek, \& Mylander, 2008; Napoli \& Sutton-Spence, 2014; Newmeyer, 2000). Goldin-Meadow et al. (2008) reported an SOV bias in a task where

\footnotetext{
${ }^{1}$ Throughout this paper, we use the S,O,V notation to refer to agents, patients, and actions of simple events in both established languages and gesture/symbol tasks. This usage matches the terminology used in the typology literature and in other gesture experiments to describe gestures produced by naïve participants.

${ }^{2}$ Estimating the prevalence of word orders is complicated by the non-independence between languages in the same family. Phylogenetic analyses of the world's languages also suggest that SOV, followed by SVO, is the most common and likely historically oldest word order (Gell-Mann \& Ruhlen, 2011)
} 
PsyArxiv preprint first posted online March 3, 2017, http://osf.io/vaqk3/. The copyright holders for this preprint (which was not peer-reviewed) are the authors. It is made available under a CC-BY-NC-ND 4.0 International license.

participants used spontaneous gesture to communicate scenes depicting an animate agent acting on an inanimate patient, e.g. a boy kicking a ball (notated animate-inanimate in this paper). Participants typically gesture $B O Y B A L L K I C K$, regardless of whether their language is verbfinal like Japanese, verb-medial like English, or a verb-initial like Tagalog (Futrell et al., 2015; Gibson, Piantadosi, et al., 2013; Goldin-Meadow et al., 2008; Hall, Ferreira, \& Mayberry, 2014; Hall et al., 2013; Langus \& Nespor, 2010).

Interestingly, this effect can be significantly modulated by scene meaning, particularly the reversibility of the events, i.e. whether either character could conceivably act as the agent (referred to here as animate-animate scenes). The boy kicks the girl is as plausible as The girl kicks the boy; the same is not the case for The ball kicks the boy. Speakers of SVO and VSO languages tend to switch from SOV to SVO gestures when the patient is animate (English: Gibson, Piantadosi, et al., 2013; Hall et al., 2013; Meir, Lifshitz, Ilkbasaran, \& Padden, 2010; Russian, Tagalog, Modern Irish: Futrell et al., 2015). In SOV languages, the same animacydependence is observed when the critical event is embedded inside another clause, e.g. The grandmother said the boy kicked the girl (Gibson, Piantadosi, et al., 2013) .

Two basic classes of hypotheses have been proposed to explain this animacy/reversibility effect. The first focuses on successful communication. Under a noisy-channel framework (cf. Aylett \& Turk, 2004; Gibson, Bergen, \& Piantadosi, 2013; Jaeger, 2010; Levy, Bicknell, Slattery, \& Rayner, 2009; Piantadosi, Tily, \& Gibson, 2011, 2012; Shannon, 1949), word order results from general pressures to communicate efficiently and robustly. Semantic likelihood alone can guide meaning reconstruction in many cases, e.g. KICKING BOY BALL- but the same is not true for KICKING GIRL BOY, where either character might be the agent of the kicking event. Several research groups have proposed that SVO order is less ambiguous because the signs are placed 
PsyArxiv preprint first posted online March 3, 2017, http://osf.io/vaqk3/. The copyright holders for this preprint (which was not peer-reviewed) are the authors. It is made available under a CC-BY-NC-ND 4.0 International license.

farther apart (Gibson, Piantadosi et al. 2013; Fischer, 1975; Meir, Lifshitz, et al., 2010; Napoli \& Sutton-Spence, 2014). Specifically, Gibson et al (2013) (abbreviated GP2013 hereafter) argue that SVO order is more robust to partial deletion (i.e. messages like KICK BOY are still partially interpretable).

The second kind of explanation involves constraints that result specifically from the gesture modality. Hall and colleagues (Hall et al. 2013; abbreviated H2013) conducted a series of gesture experiments focusing on the distribution of non-SOV orders for reversible (animate-animate) events. In addition to SVO, they found an increased prevalence of $\operatorname{OSV}(\mathrm{O})$ and $\operatorname{SOSV}(\mathrm{O})$ with these scenes (a similar distribution was found by Meir et al. 2010, though not by GP2013). These orders accounted for $30 \%$ of reversible trials, and are not predicted by the noisy-channel explanation, because subject and object are adjacent, just like SOV. Instead, H2013 argue that these orders - SVO, OSV, SOSV - all avoid local animate-O-V sequences. Why should this matter?

Participants in gesture tasks often spontaneously produce body-based gestures, e.g., referring to a person in a jacket by signing JACKET on their own torso, rather than tracing a jacket in front of them. Importantly, similar gestures are used for actions, and the signer takes the role of agent: body-based THROW usually involves imitating a throwing motion, not moving as though thrown by another. H2013 hypothesize that, when using body-based gestures, participants strongly prefer that the human entity that is gestured immediately before the verb refers to the agent, not the patient. If an animate character in the patient role has just been signed, the producer is likely to be distracted or find it infelicitous to move straight on to the verb without re-establishing the agent of the event. This is purely a production constraint: the sequence feels awkward to the producer, so they avoid it. The constraint thus predicts that participants tend to 
PsyArxiv preprint first posted online March 3, 2017, http://osf.io/vaqk3/. The copyright holders for this preprint (which was not peer-reviewed) are the authors. It is made available under a CC-BY-NC-ND 4.0 International license.

avoid a local O-V sequence for reversible (animate-animate) events, producing SVO and the other observed orders that place the agent immediately before the verb. Following H2013, we will refer to this as a constraint against the potential for role conflict.

This constraint has critical implications for word order research. Gesture experiments have been taken to be informative about the underlying structure of human language and cognition (cf. Langus \& Nespor, 2010), but if the shift from SOV to SVO is due to a modality-specific production constraint then this generalization is unwarranted. Fortunately, the noisy-channel and production accounts make different predictions about how word order might be affected by changes of experimental procedure in gesture studies. If gesture sequences are primarily organized by the need to make the signal robust for communication, changing communicative pressures might change word order, but instructions that merely modify the form of particular gestures should not change the word order. On the other hand, if the shift from SOV to SVO is due to production constraints, then the reverse is true: instructions that modify communicative pressures are unlikely to have an effect, while tasks that change how body-based gestures are used may have significant effects by avoiding the critical role-conflict constraint entirely. Of course, both of these predictions depend on the assumption that it is possible to change only one of these factors at a time.

We conducted two experiments to test these hypotheses. In the first, we attempted to modify the gesture production task to address a key prediction of the noisy-channel explanation, namely that participants give SVO orders in order to convey extra information about the roles of two animate entities in an event. In the standard gesturing paradigm, some participants also spontaneously use spatial cues to mark thematic roles (H2013, GP2013). For instance, they might place the character gestures side by side in space, and then show a 'kick' gesture moving from the agent to 
PsyArxiv preprint first posted online March 3, 2017, http://osf.io/vaqk3/. The copyright holders for this preprint (which was not peer-reviewed) are the authors. It is made available under a CC-BY-NC-ND 4.0 International license.

the patient. GP2013 found (in the standard task) that this kind of spontaneous spatial 'case marking' had a strong effect on the gesture ordering: for reversible (animate-animate) events, $64 \%$ of the spatially marked trials used SOV order (compared to $14 \%$ of the non-spatiallymarked trials). However, because this evidence comes from spontaneous differences in gesturing, its value is somewhat limited. There might have been other differences between participants who chose to use case-marking and those who didn't, or between items that tended to elicit spatially distinguished gestures and ones that didn't. Thus, the present version experimentally manipulates case-marking by contrasting the standard instructions with a version explicitly instructing participants to use a case-marking system. The noisy-channel hypothesis predicts that this variant of the gesture-production method should eliminate or reduce the need for SVO orders, because it will be easier to tell subject and object apart without resorting to changing the default SOV order. On the other hand, if word orders are determined by production constraints, participants' patterns are not predicted to change so long as their gestures in the two tasks are qualitatively similar. To test this critical assumption, we therefore also code whether body-based signs are used in the same way before and after the case marking manipulation.

To foreshadow the results of Experiment 1, we find differences in word order between the standard and explicit case-marking instructions, but also a trade-off between case marking and body-based signs. This means that either noisy channel or role-conflict theories could explain the pattern of results. To clarify this pattern, we provide a second communication task that abstracts away from role conflict constraints and other features specific to the gesture modality. In Experiment 2, we provide a task involving arbitrary symbols and the same basic communicative structure of the gesture communication task. The presence or absence of an animacy effect in this second task can license conclusions about the universality of word order in ad-hoc 
PsyArxiv preprint first posted online March 3, 2017, http://osf.io/vaqk3/. The copyright holders for this preprint (which was not peer-reviewed) are the authors. It is made available under a CC-BY-NC-ND 4.0 International license.

communication, giving us a clearer window on the nature of word order biases and on the relationship between gesture and typology in spoken language.

\section{Experiment $1^{3}$}

Experiment 1 aimed to test the effects of providing an additional way to encode role information in the gesture task. After completing the standard task, participants were explicitly instructed to use spatial marking by using different hands when signing each argument in an event. We analyzed the impact of these instructions on both cue use (i.e. using spatial cues to distinguish characters, e.g. GIRL-LEFT, OLDLADY-RIGHT) and gesture form (use of body-based signs). Prior to data collection, we did not anticipate that using spatial information would result in significant changes to the kinds of gestures that participants produced; below, we discuss the blinded coding schemes developed to identify both spatial cues and body-based gestures in the gesture sequences.

Based on this cross-coding of spatial cueing (experimentally manipulated) and body-based signs (coded after the fact), we had the opportunity to test both theories of word order in gesture experiments. The critical analyses for this experiment focus on when participants use SOV vs. SVO order for reversible (animate-animate) sentences. If noisy-channel pressures drive the switch to SVO, then SOV order should be more likely on those trials where case marking is used, because the agent and patient roles have been more clearly identified, and SVO order should be more likely when spatial information is not provided (because of pressure to

\footnotetext{
${ }^{3}$ All stimuli, presentation scripts, de-identified data, and analysis scripts for Experiments 1 and 2 are available in an online repository at http://osf.io/vaqk3/
} 
PsyArxiv preprint first posted online March 3, 2017, http://osf.io/vaqk3/. The copyright holders for this preprint (which was not peer-reviewed) are the authors. It is made available under a CC-BY-NC-ND 4.0 International license.

disambiguate the two animate participants). The particular form of the gestures is not predicted to have an effect. On the other hand, if the RCP constraints drive the shift, SVO order should be more likely when body-based signs create the environment for RCP production constraints to operate, i.e. when participants gesture animate characters by using themselves as the 'stand-in' for an event role. This constraint predicts that SOV orders should be produced for animateanimate sequences when participants produce gestures for animate characters away from the body, because the gesturer experiences no role conflict and so no pressure to switch away from the default SOV order.

\section{Method}

Participants 36 native English speakers from the Boston area participated for payment (average age 33 years, 20 female). 13 additional participants were excluded because they did not understand the gesture instructions/did not produce interpretable gesture sequences (7), because they did not complete the entire experiment (2), or because of experimenter error in administering the task (4).

Materials The stimuli consisted of 24 animated vignettes shown on a computer screen (created by K. Brink, GP2013). The full list of vignettes is shown in Table 1, and stills from example transitive and intransitive vignettes are shown in Figure 3. Sixteen vignettes depicted transitive events (e.g., kick, lift; a total of 8 unique verbs), and eight control scenes depicted intransitive events (e.g., tumble, roll; 4 unique verbs). Of the transitive events, half involved humans as both the agent and the patient, and were thus potentially reversible (e.g., boy kicks girl can be reversed to girl kicks boy). The other half had human agents and inanimate patients. In total, there were four different animate characters (boy, girl, fireman, old lady) and four inanimate objects (star, 
PsyArxiv preprint first posted online March 3, 2017, http://osf.io/vaqk3/. The copyright holders for this preprint (which was not peer-reviewed) are the authors. It is made available under a CC-BY-NC-ND 4.0 International license.

(a)

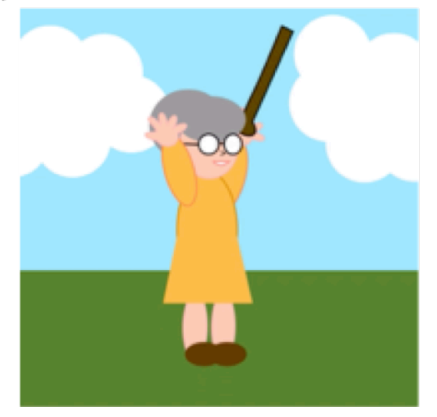

(b)

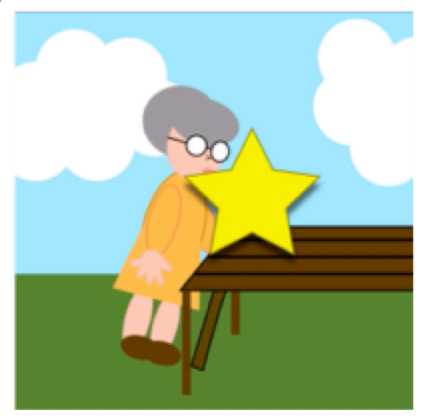

(c)

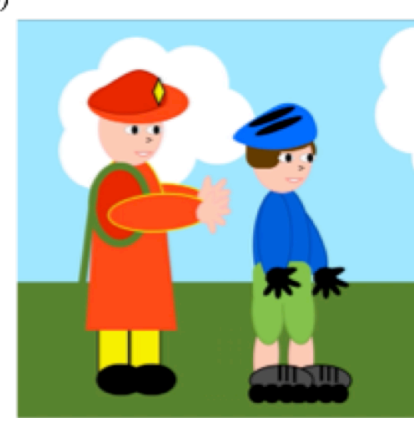

Figure 1. Three examples of stills from the animated event vignettes used in the experiment. (a) Old lady jumps Intransitive (b) Old lady kisses star - Transitive, inanimate patient (c) Fireman pushes boy - Transitive, animate patient.

Intransitive events Animate-inanimate events Animate-animate events

\begin{tabular}{lll}
\hline Old lady jumps & Boy kicks ball & Fireman kicks girl \\
Star jumps & Girl pushes car & Fireman pushes boy \\
Boy rolls & Old lady elbows ball & Girl elbows old lady \\
Ball rolls & Girl rubs heart & Old lady rubs fireman \\
Fireman falls & Fireman throws star & Girl throws old lady \\
Heart falls & Old lady kisses star & Girl kisses boy \\
Girl tumbles & Boy pokes heart & Old lady pokes fireman \\
Car tumbles & Fireman lifts car & Boy lifts girl
\end{tabular}

Table 1. A full list of the event vignettes used in the communication tasks in Experiments 1-2.

heart, car, ball) used among the 24 stimuli; Two random orderings of these stimuli were created for the experiment, with an additional two vignettes included as warm-up trials.

Procedure. The experiment consisted of three components: a verbal task and two gesture tasks. The exact text of instructions given before each task is shown in Figure 2. In the verbal task, participants were simply asked to verbally describe each vignette in a simple sentence. In the 
PsyArxiv preprint first posted online March 3, 2017, http://osf.io/vaqk3/. The copyright holders for this preprint (which was not peer-reviewed) are the authors. It is made available under a CC-BY-NC-ND 4.0 International license.

first (standard) gesture task, they watched the vignettes again and were asked to act out each

event using only gestures and no speech. No specific instructions were given about the types of gestures that participants should use, and the experimenter did not demonstrate any sample gestures or refer to event components like 'subject' or 'agent'. In the second gesture task, participants were instructed to include spatial information in their gestures as they went through the vignettes. Again, the instructions did not include mention of thematic roles: participants were simply told that they should use one hand when they need to gesture about a "person or thing", and use the other hand whenever they needed to gesture "another person or thing".

\section{Introduction:}

You are going to watch a series of 24 short videos on the screen. Your job is to explain each scene to a person who can't see the screen so that they would know what's going on.

Verbal Description Task:

We're first going to start with the verbal description part. After each video, tell me what happened in that movie with one sentence.

Gesture Task 1:

Now you are going to watch the same videos over again. This time, describe what happened using your hands. This means you are not allowed to say anything out loud, just like playing charades. Please make sure you are detailed enough so that someone who can't see the video would know what happened. Try not to talk or mouth along with your gestures.

\section{Gesture Task 2:}

In this part, you're going to watch the same slides over again, but this time I would like you to include location information in your gestures. So, whenever you need to mention an object or person, mark its location using one hand. And to mark another object or person, use the other hand. Again, please make sure you are detailed enough so that someone who can't see the video would know what happened, and try not to talk or mouth along with your gestures.

Figure 2. Instructions given to participants before each phase of the description task 
PsyArxiv preprint first posted online March 3, 2017, http://osf.io/vaqk3/. The copyright holders for this preprint (which was not peer-reviewed) are the authors. It is made available under a CC-BY-NC-ND 4.0 International license.

\section{Coding}

All responses were filmed and independently coded by two of a group of four trained coders, including at least one coder blind to condition. For blind coding, the videos were clipped into sequences of four trials from either the first or second gesture task, and then interleaved in a random order. Responses to each scene were coded to reflect the order of gestures referring to the agent, patient, and action (i.e. S, O, V). A series of consecutive gestures referring to one concept were coded as a single instance (e.g., a sequence GLASSES-CANE-BUN all referring to the old lady might be coded as an S gesture). Participants occasionally paused or re-started their gesture sequences, in which case their final continuous gesture sequence was analyzed. Re-starts were coded as any instance where the participants put their hands down and stopped gesturing for more than two seconds, or waved their hands to signal a 're-do'.

1148 gesture sequences whose content could be determined (plus 4 illegible sequences) were produced by the 36 participants. On the majority ( 833 items, $73 \%$ ) of trials, participants' responses consisted of exactly one expression of $\mathrm{S}, \mathrm{O}$, and $\mathrm{V}$ in some order. However, in some cases they either omitted a referent (e.g. VO), included multiple repetitions (e.g., SOSV) or produced simultaneous gestures (e.g., simultaneous SO followed by V). In order to code these remaining items, we asked whether the response a participant produced could be categorized as either separating or not separating the subject and object with a verb. Thus, wherever possible the remaining utterances were classified as either SVO-type (verb medial, e.g., SVO/OVS) or SOV-type (non verb-medial, e.g., OSV, VOS, etc.). To do this, we restricted responses to the final three gestures produced on each trial, and checked whether this response included all three referent types in a code-able order. For instance, simultaneous $\mathrm{S}$ and $\mathrm{O}$ followed by $\mathrm{V}$ would be coded as SOV-type, whereas $\mathrm{S}$ followed by simultaneous $\mathrm{O}$ and $\mathrm{V}$ would be left unclassified. 
PsyArxiv preprint first posted online March 3, 2017, http://osf.io/vaqk3/. The copyright holders for this preprint (which was not peer-reviewed) are the authors. It is made available under a CC-BY-NC-ND 4.0 International license.

Responses consisting of only one or two gestures were left unclassified since the position of the two nouns relative to a verb could not be defined ${ }^{4}$. Overall, this process allowed for the inclusion of an additional 124 items for a total of 957 items in the analysis; none of the effects reported below are significantly affected by the inclusion or exclusion of these 124 items. Inter-coder reliability was calculated over both raw gesture codings and over final classifications (SOV-type, SVO-type, Unclassified). On the raw codings, coders agreed on $78 \%$ of trials (Cohen's kappa $=$ 0.72), and on the final classifications coders agreed on $88 \%$ of trials (Cohen's kappa $=0.80)$. All disagreements were resolved by discussion with a third member of the coder group who remained blind to condition.

All responses were also coded to reflect whether spatial information was used to distinguish subject and object. In addition to using the instructed marking system (and consistent with prior reports), participants spontaneously developed alternative spatial systems during both gesture tasks. During the process of coding, we discovered a number of strategies used to convey thematic role information with spatial cues. Because this variety of case marking strategies was discovered during the coding process described above, a new coder who was blind to the hypothesis and condition re-coded $10 \%$ of the data (4 participants) for spatial/case marking information. Agreement was very high $(92 \%$, Cohen’s kappa $=0.84)$ and remaining disagreements were resolved by discussion among blind-to-condition coders. Responses were coded as including spatial/case marking information if participants added a specific marker (e.g.,

\footnotetext{
${ }^{4}$ This final-three-gesture coding was designed to capture the relevant information about each gesture sequence (position of $\mathrm{V}$ relative to $\mathrm{S} / \mathrm{O}$ ) in a straightforward way. 133 gesture sequences were produced that were longer than 3 gestures (e.g. SOSOV), of which 97 could be classified under this metric as SVO-type or SOV-type. Because these longer sequences typically consisted of repetitions of $\mathrm{S}$ and $\mathrm{O}$ at the beginning of the sequence, the truncation usually either reflected the overall order (e.g. SOSOV $->\mathrm{SOV}$ ) or resulted in the 'unclassified' coding for marginal SVO sequences - those where one of either $\mathrm{S}$ or $\mathrm{O}$ appears both before and after a verb, but the other has a unique ordering (e.g. SVOV -> VOV). Of the 97 successfully classified items, only three tokens appear potentially questionable under our classification scheme (two instances of SOVSVO classified as SVO-type; one instance of OVOSV classified as SOV-type).
} 
PsyArxiv preprint first posted online March 3, 2017, http://osf.io/vaqk3/. The copyright holders for this preprint (which was not peer-reviewed) are the authors. It is made available under a CC-BY-NC-ND 4.0 International license.

1 finger or 2 fingers following a noun sign) or produced the sign for an agent or patient (at least one) in a specific location away from their neutral signing posture, by gesturing to a new location in front of them or by shifting their torso (following possibilities for spatial distinguishing of referents in American Sign Language and International Sign pidgin, Lillo-Martin \& Klima, 1990; McKee \& Napier, 2002). Thus, we did not code incidental differences in location due to the production of particular signs (e.g., HELMET, which was typically gestured in an upper part of the signing space vs. ROLLERSKATES, which were typically gestured in a lower part of the space) as 'spatial information'.

Finally, each gesture element ( $\mathrm{S}, \mathrm{O}$, or $\mathrm{V}$ sequence) in a response sequence was coded as either body-based or not body-based. In both tasks, body-based signing and spatial information were not mutually exclusive - gestures sequences could include either, both, or neither. For nouns, gestures were coded as body-based if the distinctive features of a person (e.g. accessories, hairstyles, postures) were located in the appropriate place on the signer's own body. If gestures for people were placed in the signing space in front of the participant, or indicated in a way that didn't place a character's feature in the analogous place on the participant's own body, the gesture was coded as not body based. Three example gesture glosses referring to the Old Lady character are given below:

(5) GLASSES (both hands to face) - Body based, no spatial information

(6) LEFT HAND WAVE + GLASSES (left hand to face) - Body based, spatial information

(7) LEFT HAND FINGERS 'WALK' + GLASSES (fingers form circle near table) - Spatial information, not body based. 
PsyArxiv preprint first posted online March 3, 2017, http://osf.io/vaqk3/. The copyright holders for this preprint (which was not peer-reviewed) are the authors. It is made available under a CC-BY-NC-ND 4.0 International license.

$30 \%$ of responses were coded for the body-based judgments by two coders, one blind to condition (as described above); inter-coder agreement was very high (96\%, Cohen's kappa $=$ $0.865)$.

\section{Results}

\section{Word Order}

We first evaluated whether the case marking instructions led to a general shift in the distribution of word orders produced by participants (Figure 3). We focus on the classification of gesture orders as either SVO-type (any verb-medial order, i.e. those which order one event argument before the verb and one after) or SOV-type (any verb non-medial order, wit both arguments on the same side of the verb, like SOV.) The classification of gesture orders is described above, but the majority of items in these categories consist of exactly SOV and SVO orders, and results show the same qualitative pattern if only those orders are evaluated.

During the first gesture task, results for both inanimate and animate patients closely paralleled previous findings (Goldin-Meadow et al., 2008, Gibson et al., 2013). This ordering difference was statistically significant in a two-tailed, mixed-effects logistic regression that included random participant slopes and item intercepts, $\mathrm{X}^{2}=22.56, \mathrm{df}=4, \mathrm{p}<.001 .^{5}$ In the second task (after instruction to use spatial information to mark the arguments of the events), participants

\footnotetext{
${ }^{5}$ The animacy varied within subjects, but not within items. The model with maximal random effects can thus be expressed in R as glmer(WordOrder Object.Type + (Object.Type|Subject) + (1|Sentence), family="binomial"). This kind of analysis is used for all remaining effects reported in the experiment: the model with full random participant slopes and intercept and item intercepts is reported unless it fails to converge, in which case random effect factors are removed until convergence is reached. Full details of all analyses are available in the project repository, http://osf.io/vaqk3/
} 
PsyArxiv preprint first posted online March 3, 2017, http://osf.io/vaqk3/. The copyright holders for this preprint (which was not peer-reviewed) are the authors. It is made available under a CC-BY-NC-ND 4.0 International license.

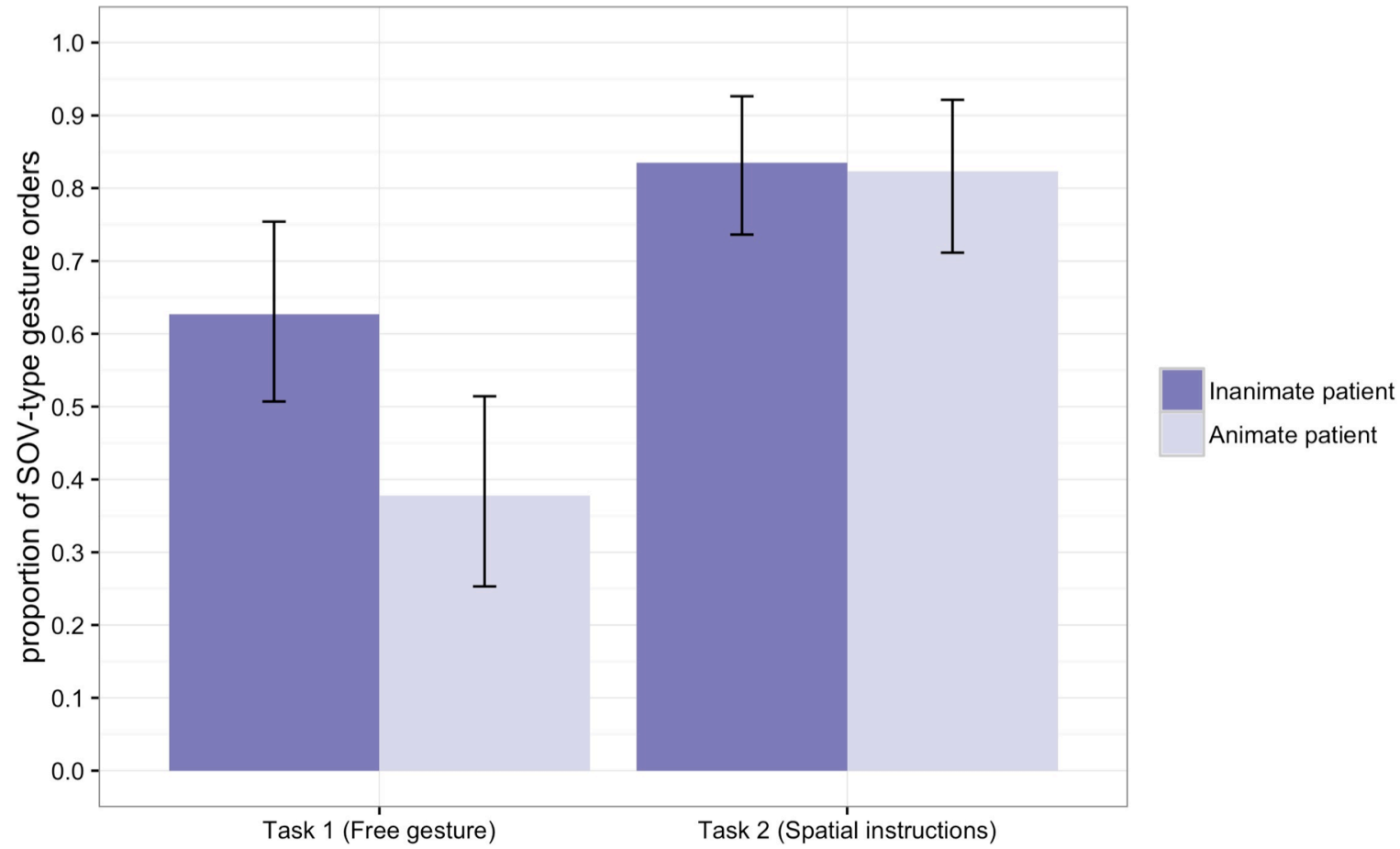

Figure 3. Proportion of gesture sequences using SOV-type orders in the first (free gesture) and second (spatial/case marking instructions) gesture tasks. Error bars represent bootstrapped $95 \%$ confidence intervals by participant.

tended to produce SOV-type sequences regardless of animacy. The difference in verb-final gestures for animate and inanimate patients was not statistically significant for this second task $\left(X^{2}=0.07, d f=6, p=.79\right)$. Analyzing the two gesture tasks together, we found a significant task by animacy interaction $\left(X^{2}=16.91, \mathrm{df}=6, \mathrm{p}<.001\right)$, as well as main effects of animacy $\left(\mathrm{X}^{2}=\right.$ 18.22, $\mathrm{df}=5, \mathrm{p}<.001)$ and gesture task $\left(\mathrm{X}^{2}=184.1, \mathrm{df}=5, \mathrm{p}<.001\right)$.

\section{Spatial cues and body-based signs}

We next evaluated the two possible mechanisms for word order, namely (1) whether participants produced spatially coded gestures on individual trials and (2) whether they used body-based gestures. As noted above, participants varied in their use of spatial information during both tasks. Likewise, we found body-based signs used in both of our gesture tasks. Our experimental manipulation can only provide unique support for the noisy-channel model if the instructions did 
PsyArxiv preprint first posted online March 3, 2017, http://osf.io/vaqk3/. The copyright holders for this preprint (which was not peer-reviewed) are the authors. It is made available under a CC-BY-NC-ND 4.0 International license.

not also lead participants to avoid body-based gestures. H2013 describe the potential for role conflict (RCP) as occurring when a gesturer has just taken on the role of an animate patient (with a body-based sign) and is planning a verb gesture in which the will take on the role of the agent (another body-based sign). Our critical classification of body-based gesture sequence was therefore whether or not a particular gesture sequence contained both a body-based verb sign and a body-based sign for an animate patient (in any order). We refer to responses meeting these conditions as gestures with RCP.

Our intended 'case marking' manipulation was successful: after receiving instructions before the second gesture task, participants produced many more gestures with spatial information overall $\left(X^{2}=43.05, \mathrm{df}=7, \mathrm{p}<.001\right)$. There was also a small but significant interaction between task and animacy $\left(\mathrm{X}^{2}=6.29, \mathrm{df}=8, \mathrm{p}<.05\right)$ : after instruction, participants' use of spatial information increased somewhat more for animate trials in particular, a pattern which is loosely in line with the predictions of the noisy channel theories (more spatial information provided for more confusable referents). In addition, we also saw significant changes in the forms of the gestures people produced. Within animate-animate trials, RCP (body-based) gestures declined significantly from the first gesture task to the second $\left(\mathrm{X}^{2}=22.24, \mathrm{df}=8, \mathrm{p}<.001\right)$. (RCP occurs only when the patient is animate, so the value for inanimate patients in both tasks is $0 \%$.)

\section{Relationship between word order and gesture features}


PsyArxiv preprint first posted online March 3, 2017, http://osf.io/vaqk3/. The copyright holders for this preprint (which was not peer-reviewed) are the authors. It is made available under a CC-BY-NC-ND 4.0 International license.

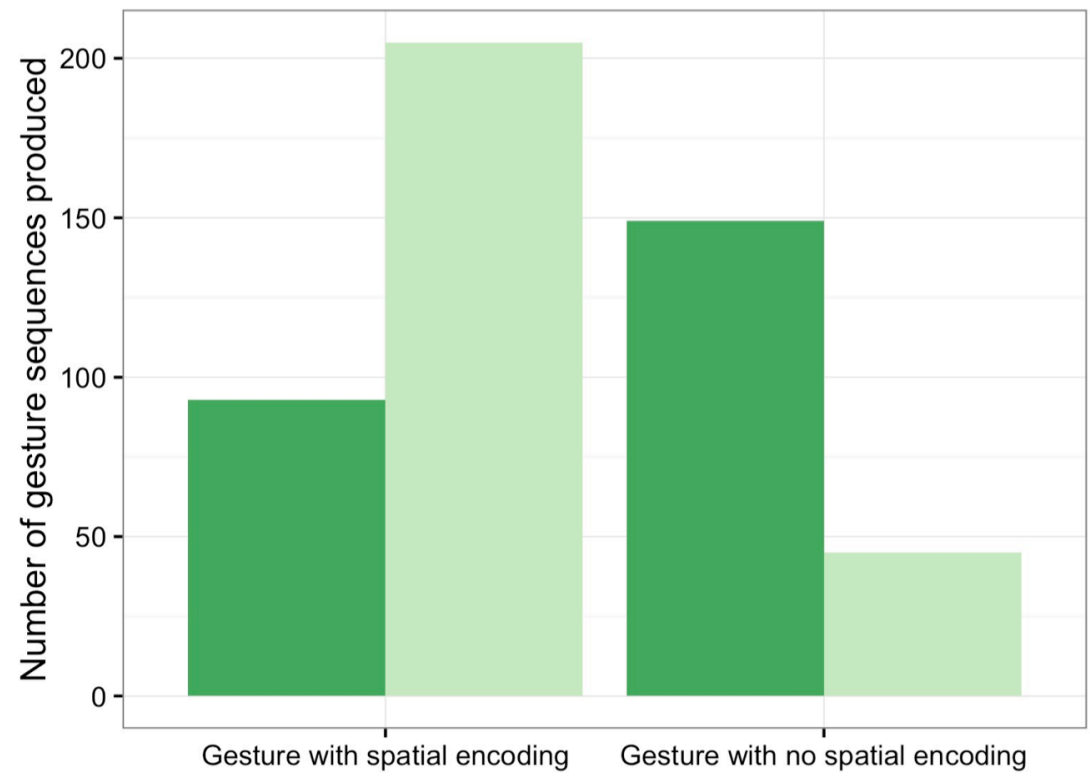

Gesture order with RCP Gesture order without RCP

Figure 4: Histogram of gesture trials (across both tasks) with role conflict potential (body-based gestures) and spatial encoding of agents and/or patients.

Word order patterns changed between the first and second gesture tasks, and so did both of our gesture features of interest. While we cannot establish a causal relationship between these features, we nevertheless attempt to describe the relationships between spatial coding, role conflict, and word order. To do this, we limit the remaining analyses to the animate trials since the primary effects in both theories apply to these kinds of events in particular.

Use of spatial information in gesture sequences was closely related to gesture order, as found anecdotally in GP2013). Across both experiments, participants were more likely to gesture in an SOV-type order when they also used spatial information (Task $1, X^{2}=18.50, \mathrm{df}=8, \mathrm{p}<0.001$; Task $\left.2, \mathrm{X}^{2}=7.55, \mathrm{df}=8, \mathrm{p}<0.01\right)$. There was no interaction between task and case marking on the gesture order used $\left(\mathrm{X}^{2}=0.05, \mathrm{df}=8, \mathrm{p}=0.82\right)$. The presence of $\mathrm{RCP}$ (body-based gesture) was also closely related to gesture order: participants were less likely to use an SOV-type order for animate-animate events when role conflict potential was present. This difference was significant in both the free gesture task $\left(\mathrm{X}^{2}=6.47, \mathrm{df}=6, \mathrm{p}<.001\right)$, and in the case-marking instruction task 
PsyArxiv preprint first posted online March 3, 2017, http://osf.io/vaqk3/. The copyright holders for this preprint (which was not peer-reviewed) are the authors. It is made available under a CC-BY-NC-ND 4.0 International license.

$\left(\mathrm{X}^{2}=3.98, \mathrm{df}=8, \mathrm{p}<0.05\right)$; with an additional significant interaction between role conflict and $\operatorname{task}\left(\mathrm{X}^{2}=3.97, \mathrm{df}=8, \mathrm{p}<0.05\right)$

Both of these patterns were in the expected directions: absence of role-conflict and presence of spatial information were both associated with SOV-type orders for animate-animate trials. To conclude, we explored the contingency between RCP and spatial coding. These features were highly related: the presence of RCP and spatial coding were negatively correlated $\left(\mathrm{X}^{2}=19.36\right.$, $\mathrm{df}=6, \mathrm{p}<.001)$. In other words, when people produced gestures with RCP (i.e. body based gestures), they were less likely to use spatial information to differentiate event arguments, and vice versa (Figure 4).

\section{Discussion}

In this experiment, we first replicated the critical gesture pattern (Gesture Task 1): participants shifted from primarily SOV-type orders to primarily SVO-type orders when describing reversible events (events with two animate characters, where either could be the agent or patient of the action). Previously, GP2013 argued that this pattern reflects a tendency to produce sequences that facilitate recovering the correct meaning under noisy conditions. For nonreversible events where patients are inanimate, the action could only occur in one plausible way - a boy can kick a ball, but a ball cannot kick a boy, so the order of gestures is not critical to interpreting a sequence like BOY BALL KICK. However, this is not the case for reversible events (a girl can kick a boy and a boy can kick a girl). SVO order is argued to improve recoverability because a mis-hearing (e.g. KICK BOY), can maintain the relative order of the agent and patient, which a comprehender can use as a cue to the intended meaning. 
PsyArxiv preprint first posted online March 3, 2017, http://osf.io/vaqk3/. The copyright holders for this preprint (which was not peer-reviewed) are the authors. It is made available under a CC-BY-NC-ND 4.0 International license.

Our goal in the case-marking task was to tease apart the noisy channel and role conflict/production explanations by encouraging participants to add spatial details to gestures without materially affecting the general form of participants' gestures. However, we were unsuccessful: the instructions in fact led to significant changes in how participants gestured animate characters. In addition to using just one hand, participants often gave non body-based signs, e.g. gesturing $\mathrm{BOY}$ as an inverted V 'walking' on the table rather than as a bicycle helmet on their own head. By necessity, the gesture task with specific instructions (spatial coding) had to be administered after the no-instructions gesture task, raising the possibility that some of the changes we saw were due to practice effects or demand characteristics of being asked to communicate the same scenes a third time (i.e. after describing them in words and then in unconstrained gesture.) However, we saw significant variance in both word order and gesture style across both tasks, creating an opportunity to evaluate the predictions of both noisy channel and role-conflict hypotheses.

Critically, we found that using spatial information - (which would increase the use of SOV-type orders under the noisy channel explanation)- and avoiding RCP (which would increase the use of SOV-type orders under the production explanation)- were confounded with one another. That is, SVO-type gestures for animate-animate scenes were produced most often when participants used body-based gestures and didn't distinguish between characters with spatial information. When they used spatial cueing of event roles, they also used fewer body-based gestures and tended to produce SOV-type orders. Thus, these data do not disambiguate between the explanations offered by GP2013 and H2013. Of course, it is also possible that both proposed mechanisms were acting in concert to produce the observed patterns. That is, it might both be the case that gesturing tasks have particular constraints that affect ordering, and that general 
PsyArxiv preprint first posted online March 3, 2017, http://osf.io/vaqk3/. The copyright holders for this preprint (which was not peer-reviewed) are the authors. It is made available under a CC-BY-NC-ND 4.0 International license.

communicative constraints (following a noisy-channel model) influence ordering as well. To distinguish between these possibilities, Experiment 2 provides a paradigm that eliminates the choice between body-based and other gestures, by moving away from the gesture modality entirely.

\section{Experiment 2}

While the spontaneous gesture paradigm has been a key tool for testing theories of word order over the past several years, Goldin-Meadow et al. (2008) also included a second experiment: a picture-creation task in which participants were asked to stack up a series of partial pictures printed on clear plastic (each corresponding to an element of an animate-inanimate event) to form the entire scene. As with the gesture task, participants from many different language groups showed an SOV ordering bias, despite the fact that this task did not involve a communicative partner. As with the gesture paradigm, this study used only events with animate agents and inanimate patients. If the noisy-channel explanation of this task is correct, then the same patterns (shift to SVO-type orders for animate-animate scenes) should result for picture construction as well as gesture. On the other hand, if these orders are a specific byproduct of gesture, we might see a replication of the basic SOV ordering effect, but no effect of the animacy of the patient.

However, a null effect for animacy in the exact picture task implemented in Goldin-Meadow et al. might be vulnerable to some of the same critiques as the gesture task. Because of the particulars of the picture-stacking task, the depictions chosen for each event element might conceivably have some ordering effects (analogous to the role of body-based signs). In particular, it is very difficult to come up with a depiction of a verb that doesn't reference any of its arguments (e.g. kicking, without showing a person or at least a foot). Goldin-Meadow et al. 
PsyArxiv preprint first posted online March 3, 2017, http://osf.io/vaqk3/. The copyright holders for this preprint (which was not peer-reviewed) are the authors. It is made available under a CC-BY-NC-ND 4.0 International license.

solved this problem by using arrows and other symbols to show relations between arguments, and this difference (symbolic arrows vs. realistic agents \& patients) could have influenced the orders that people choose to assemble the pictures.

Instead, we use a purely symbolic task, in which participants must pass novel, non-iconic symbols (which they have been taught represent specific subjects, objects, and verbs) to a communication partner. This is implemented as an online task where participants click on symbols one at a time out of an array to send them to a cartoon 'listener'. Because symbols are randomly placed and meanings are randomly assigned, any patterns found in the orders participants choose to send the symbols must be due solely to their meanings. While the task is necessarily somewhat more artificial than acting out a scene for another person (a relatively familiar game in many cultures), it provides a strong test of the noisy channel explanation for gesture ordering by retaining all of the semantic and informational details believed to be relevant while replacing any influence from modality-specific features with a fully arbitrary channel. Thus, if an animacy effect (an increase in SVO-type ordering for animate-animate scenes) is observed, it will be because the difference in meaning leads to a change in ordering preferences, consistent with the noisy channel explanation. Alternately if we observe no difference between animate-animate and animate-inanimate scenes, we should conclude that the ordering effects are specific to the spontaneous gesture task, due to modality-specific production constraints rather than reflective of universal communication pressures.

\section{Methods}

Participants 292 participants participated on Amazon's Mechanical Turk over the course of seven days. All adults accessed the survey from United States IP addresses and self-reported as 
PsyArxiv preprint first posted online March 3, 2017, http://osf.io/vaqk3/. The copyright holders for this preprint (which was not peer-reviewed) are the authors. It is made available under a CC-BY-NC-ND 4.0 International license.

native English speakers. Participants gave informed consent in accordance with MIT IRB protocols, and were paid $\$ 5$ for their participation in line with the lab's typical Mechanical Turk pay rates.

Materials A subset of the event items from Experiment 1 was used for the online task. We reduced the total number of arguments (old lady, fireman, girl, boy, ball, heart, car), and verbs (tumbling, pushing, rolling, kissing, lifting, kicking, throwing, rubbing, elbowing) to 16 total, to decrease the memory burden and display space required for the symbols. There were four filler (intransitive) trials, and seven each of animate-animate and animate-inanimate transitive scenes. All participants saw all items in the experiment (a within-subjects design), presented in a random order generated for each participant. Figure 5 shows the basic setup of the task (described below), including the sixteen symbols representing subjects, objects, and verbs in the task. The symbols were chosen to be sufficiently different from one another to be recognizable, without obvious iconic interpretations. For each participant, each symbol's meaning was randomly assigned. The online task was designed and presented on Mechanical Turk using a combination of JsPsych (de Leeuw, 2015) and PsiTurk (Gureckis et al., 2015); all code for the presentation script is available at https://github.com/mekline/GlyphGrid.

Procedure The task was framed as a game involving communicating with an alien character who communicated in symbols ('Upwilaah'). Participants performed two sub-tasks, each taking approximately 15 minutes to complete. In the first half, they memorized the meanings of 16 symbols, and in the second half they watched the action scenes and clicked symbols to 'send' them to their partner. To discourage participants from writing down or otherwise recording the symbol stimuli (which was reported by many participants during pilot testing), the symbols were first presented on a fixed schedule one at a time with their meaning printed underneath (each for 
PsyArxiv preprint first posted online March 3, 2017, http://osf.io/vaqk3/. The copyright holders for this preprint (which was not peer-reviewed) are the authors. It is made available under a CC-BY-NC-ND 4.0 International license.

3 seconds). Participants could elect to view the whole sequence multiple times until they were ready to move onto the quiz. For the quiz, participants saw the array of symbols in a random order, and were asked, for each meaning, to select the appropriate symbol. Participants received feedback on every trial; if they were incorrect they were shown the correct symbol before moving to the next question. Participants repeated the quiz (with the option to go back to the symbol-display sequence) until they had successfully labeled all the meanings with symbols. The median participant took two rounds through the symbol presentation and quiz to learn all 16 meanings.

After learning the symbol-meaning pairings, participants moved onto the main communication task. The instructions given for the communication task were as follows:

In this part, you will be giving symbols to Upwilaah so that he can guess what is happening. First watch the movie. After the movie is over, give Upwilaah the symbols that best describe what happened in the movie. Upwilaah will tell you how many symbols you need per movie. Remember - your goal is to answer accurately and quickly. The screen will turn green when it's time for you to answer, and then it will turn orange and red if too much time is passing. Try to keep the screen green throughout the task.

In pilot testing, we discovered that allowing unconstrained time for responses lead to nearuniversal SVO orderings, unsurprising since participants were English speakers. To encourage rapid responses, the background of the test window turned green once the movie ended, then orange (after 5 seconds) and then red (after 10 seconds) The time passed since the trial start was also displayed for participants above the block of symbols. 
PsyArxiv preprint first posted online March 3, 2017, http://osf.io/vaqk3/. The copyright holders for this preprint (which was not peer-reviewed) are the authors. It is made available under a CC-BY-NC-ND 4.0 International license.
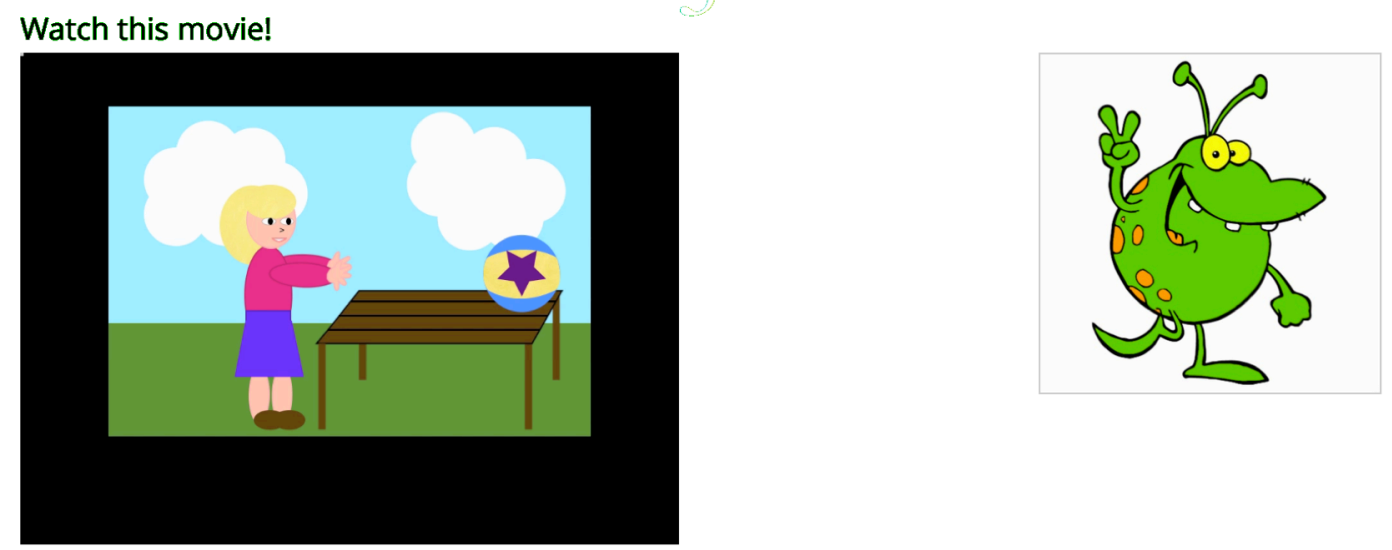

Click on the appropriate symbols so that Upwilaah knows what you are looking at. Then press DONE. Upwilaah wants 3 symbols.

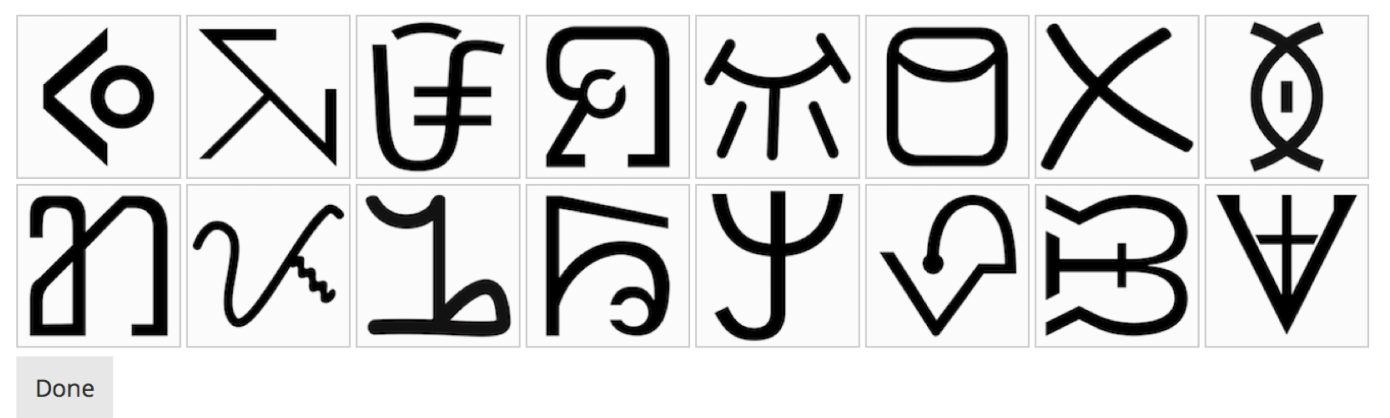

Figure 5: The visual layout of the online symbol-passing task. Participants first saw the movie play in the left-hand corner; once it had finished they could click on up to three symbols from the array (re-shuffled on every trial) to sent to the alien in his 'listening box'

The experiment began with two static images and then two practice trials (see Experiment 1) to familiarize participants with the setup, followed by the 18 test trials. On each trial, the movie played automatically; controls were disabled so that participants could not reload or replay the video. The symbols were randomly ordered in the array each time so that participants would need to search again on every trial, to reduce variance resulting from certain symbols being nearer/farther for participants to reach. Clicking on a symbol cause it to move up to the alien picture; each successive symbol covered the previous one entirely. 
PsyArxiv preprint first posted online March 3, 2017, http://osf.io/vaqk3/. The copyright holders for this preprint (which was not peer-reviewed) are the authors. It is made available under a CC-BY-NC-ND 4.0 International license.

After the task was complete, participants were debriefed about the meaning of the study; in addition, we checked again for participants who were writing down or otherwise referencing the symbols during the task by explicitly asking them to report this; 56 people reported writing down the symbols or otherwise recording them are were excluded from all analyses below ${ }^{6}$.

\section{Coding}

In contrast to the gesture task, participants' responses could be unambiguously identified as a string of symbols, e.g. SSVO. Because the goal of this study was to explore the distribution of SVO- and SOV-type ordering, rather than the full set of possible orders, we used a simplification procedure designed to convert participants' raw responses into one of the 6 basic word orders, which could then be classified as either verb medial (SVO-type) or not (SOV-type). To do this, we saved the first three unique symbols passed by participants on transitive event trials, e.g. $\mathrm{SSVO} \rightarrow \mathrm{SVO}$ or SOSV $\rightarrow$ SOV. Because many participants gave incorrect symbols on some responses, we allowed for a 'relaxed' coding scheme in which a single symbol that did not match any of the three elements of the event could stand in for the missing symbol, e.g. SXO $\rightarrow$ SVO. If participants gave more than one incorrect symbol on a trial (e.g. OXY) or did not use all three event components (OOS), the trial was dropped from analysis. The full simplification algorithm is available at http://www.github.com/GlyphGrid, as with Experiment 1, the majority of codable responses were exactly SOV or SVO, with a minority of rare orders dealt with as just described. This procedure resulted in a total of 2708 responses (out of 3262 raw responses to transitive trials from non-cheating participants).

\footnotetext{
${ }^{6}$ Three questions were asked to all participants: Did you copy down the symbols on a sheet of paper? (Y/N) Did you take a screen shot or take a picture of the screen to help you during the task? (Y/N) Did you memorize and remember the symbols on your own? (Y/N).
} 
PsyArxiv preprint first posted online March 3, 2017, http://osf.io/vaqk3/. The copyright holders for this preprint (which was not peer-reviewed) are the authors. It is made available under a CC-BY-NC-ND 4.0 International license

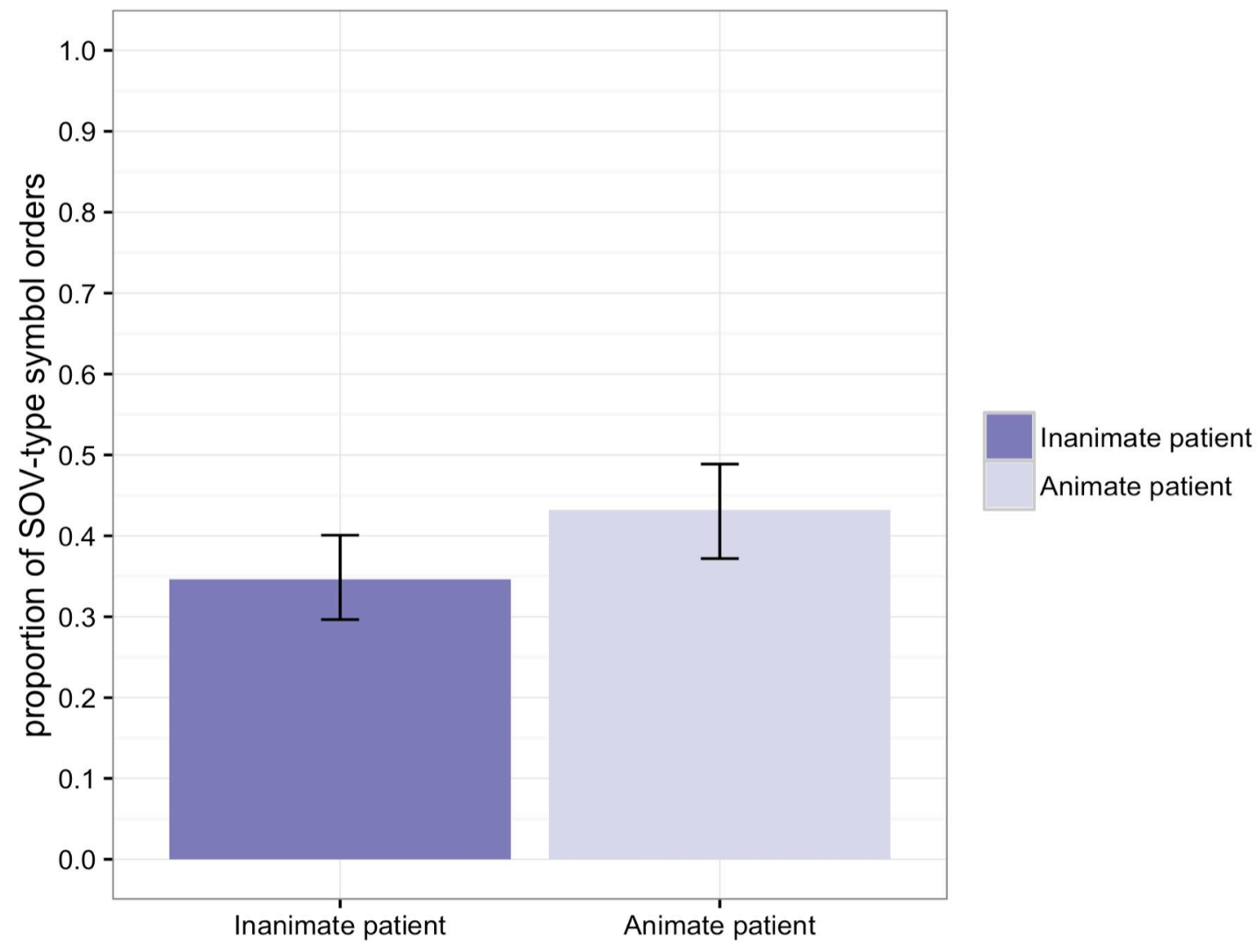

Figure 6: SOV-type orders given by participants using at least one non-SVO order during the symbol-passing task.

\section{Results}

Despite encouragement to give rapid responses, the modal order used by participants was SVO; $47 \%$ of participants only ever responded using an SVO order. Except for the large number of SVO-type responses, the overall distribution of orders was similar to that found in many gesture studies: after SVO (77\%), the next most frequent order was SOV (11\%), followed by OSV and OVS $(5 \%, 4 \%)$ with rare instances of VOS and VSO (1\% each). However, there was no significant difference in the orders participants chose for events with animate or inanimate participants $\left(\mathrm{X}^{2}=0.439, \mathrm{df}=8, \mathrm{p}=0.51\right)$. Even excluding participants who only gave $\mathrm{SVO}$ answers, the predicted pattern is not seen, in fact there are slightly more SVO-type responses to animate- 
PsyArxiv preprint first posted online March 3, 2017, http://osf.io/vaqk3/. The copyright holders for this preprint (which was not peer-reviewed) are the authors. It is made available under a CC-BY-NC-ND 4.0 International license.

inanimate scenes, not less $\left(\mathrm{X}^{2}=4.37, \mathrm{df}=8, \mathrm{p}<0.05\right)$. The proportion of SVO-type responses given the subset of participants who used non-native orders is shown in Figure 6.

\section{Discussion}

In contrast to the predictions of the noisy channel model explanation for gesture orders, and matching H2013's gesture-specific (RCP) production account, we saw no difference in the proportion of SVO and SOV-type orders used to communicate animate-animate and animateinanimate events when the modality was a series of non-iconic written symbols. While many participants in this online task simply gave their native language word order (SVO) on all trials, the subset of participants who did use multiple orders conformed to the general ordering phenomena seen across many modalities: SOV-type orders were by far the most frequent nonnative order given. However, the predicted effect of patient animacy was not found: in fact there was a small difference in the opposite direction, namely, participants were slightly more likely to use SOV-type order when the patient of the event was an animate character.

\section{General Discussion}

A robust pattern of effects has emerged concerning the word orders that participants (across a variety of language background) use when gesturing simple transitive events. When agents are animate and patients are inanimate, people tend to use SOV orders regardless of their native language, but when both agents and patients are animate, people tend to switch gestures to SVO order. While these results have been replicated several times, the explanations given have been in significant dispute. Many of these explanations have relied on the assumption that gesturing by naïve participants reflects modality-independent word order preferences. (GP2013; Langus \& Nespor, 2010; Meir et al., 2017, 2010) However, it has also been pointed out that the gesture task 
PsyArxiv preprint first posted online March 3, 2017, http://osf.io/vaqk3/. The copyright holders for this preprint (which was not peer-reviewed) are the authors. It is made available under a CC-BY-NC-ND 4.0 International license.

itself may present specific production constraints for participants in the task (H2013). Here, we present two experiments that challenge the noisy-channel communication explanation for gesture ordering. In the first experiment, we added a 'case-marking' manipulation that was intended to affect only the ambiguity of the gestures, by encouraging participants to spatially differentiate agents and patients. Under this manipulation, participants tended to use SOV-type orders even for animate-animate scenes, as predicted by a noisy-channel model. However, we discovered a second mechanism that might have produced the same observed effect: the instructions also led participants to produce signs away from their own body, reducing the role-conflict production constraint proposed by H2013. To differentiate between these mechanisms, we next implemented a parallel task using non-iconic symbols instead of gestures as the communication modality. A noisy-channel model would predict this to have no effect on the word orders used, but we found a significant impact: asking participants to communicate using non-iconic written symbols entirely eliminated the animacy-dependent order pattern. Instead, while native (SVO) orders predominated across the board in the online symbol-passing task, participants produced many SOV-type orders, significantly outnumbering the other four basic word orders (OSV, OVS, VSO, VOS) but not showing the expected animacy pattern.

The goal of the present studies was to determine whether the gesture paradigm developed by Goldin-Meadow et al. (2008) should be used to make inferences about the source of basic word order typologies. For this to be the case, it has been assumed in previous studies by Gibson et al. and many others that the gesture orders produced by participants are a relatively straightforward 'translation' of the underlying order they might communicate concepts in if they were not influenced by their native language. However, gesturing is unique in at least one way as a communication task: unlike spoken language and to a greater degree than developed, 
PsyArxiv preprint first posted online March 3, 2017, http://osf.io/vaqk3/. The copyright holders for this preprint (which was not peer-reviewed) are the authors. It is made available under a CC-BY-NC-ND 4.0 International license.

grammaticalized sign languages, the gestures that people produce are usually iconic to some degree. Therefore, they sometimes express multiple meaning elements together in a coordinated way (e.g. signs for verbs that position the gesturer in the role of the agent, as described by H2013). This iconicity may produce additional constraints on production or communication; in fact, it has been proposed that the strong SOV bias in mature sign languages may be due to the visual iconicity present in manual languages (Cuxac \& Sallandre, 2007; Napoli \& SuttonSpence, 2014; Taub, 2001). These findings are also convergent with recent work by Kocab, Lam, \& Snedeker, (under review) showing a lack of SVO-type orders for inanimate-inanimate scenes, which should act like animate-animate scenes according to noisy channel explanations, but in fact show predominantly SOV-type orders as predicted by modality-specific explanations.

Studies using the gesture task have produced some novel insights into how people communicate (or attempt to communicate) in an ad-hoc system outside their native language. The crosslinguistic consistency in gesture patterns is striking and well replicated, particularly the finding that SOV order is primary or default for prototypical transitive scenes (e.g. an animate agent causing a motion or change of state in an inanimate patient). Converging evidence from existing languages and language change suggest that SOV might be a more basic order for gesturing events, but the present results show that while promising, the gesture task does not directly support this theory: it cannot be taken as an unbiased window to the underlying orders that people use to conceptualize of and communicate events. This view can account for a number of puzzling findings in the literature including differences in rare orders across datasets and diverging patterns for certain classes of verbs (producers find it odd to mention the patient of an event before a verb that describes making that object, resulting in SVO orders for animateinanimate events like GIRL BAKE CAKE, Schouwstra \& de Swart, 2014). Instead of a unbiased 
PsyArxiv preprint first posted online March 3, 2017, http://osf.io/vaqk3/. The copyright holders for this preprint (which was not peer-reviewed) are the authors. It is made available under a CC-BY-NC-ND 4.0 International license.

window into conceptual representation and communicative pressures, these gestures are the result of a process that reflects the non-linear nature of gesture, a possibly idiosyncratic set of iconic representations, and other modality-specific production constraints.

Where do these results leave noisy-channel theories of communication? The present studies attempted to test a specific model of rational production, assuming a simple noise channel that might disrupt the order of simple sentences. Noisy-channel models have been successful in predicting and explaining phenomena in natural languages at the level of phonological, lexical, and syntactic structure (Gibson, Bergen, et al., 2013; Jaeger, 2010; Levy et al., 2009; Mahowald, Fedorenko, Piantadosi, \& Gibson, 2013). The central patterns of gesture production have been taken to mean that noisy-channel models might also explain basic word order phenomena (cf. GP2013), but the present research shows that this evidence is far from unambiguous. It is possible that noisy-channel type effects do influence ad-hoc communication, even if gesture paradigms do not reveal them, or that (as is possible from the results of Experiment 1) these effects are confounded with other production constraints. The gesture task is attractive as a research tool because it is natural for participants and appears to be relatively free of nativelanguage bias, as well as being rich enough to support a wide range of hypotheses and research questions. However, that same richness has a clear downside: participants invent gestures and string them together in unexpected ways, with the potential to reach new solutions to production pressures, and combine theoretically separate aspects of the communication system in unexpected ways. Tasks like these, however, provide an important complement to studies of existing and newly developing languages as we attempt to understand the typology of word order and the role of communication effects in the design of language. Particularly for explanations of human language that draw on more general principles of robust and parsimonious 
PsyArxiv preprint first posted online March 3, 2017, http://osf.io/vaqk3/. The copyright holders for this preprint (which was not peer-reviewed) are the authors. It is made available under a CC-BY-NC-ND 4.0 International license.

communication, controlled lab studies offer the chance to test specific predictions of a theory

with a high degree of control. Careful attention to the constraints and affordances of the

particular mediums chosen when designing such ad-hoc communication experiments will make

it possible to test these theories more precisely. 
PsyArxiv preprint first posted online March 3, 2017, http://osf.io/vaqk3/. The copyright holders for this preprint (which was not peer-reviewed) are the authors. It is made available under a CC-BY-NC-ND 4.0 International license.

\section{References}

Aylett, M., \& Turk, A. (2004). The Smooth Signal Redundancy Hypothesis: A Functional Explanation for Relationships between Redundancy, Prosodic Prominence, and Duration in Spontaneous Speech. Language and Speech, 47(1), 31-56.

https://doi.org/10.1177/00238309040470010201

Cuxac, C., \& Sallandre, M.-A. (2007). Iconicity and arbitrariness in French Sign Language: highly iconic structures, degenerated iconicity and grammatic iconicity. In E. Pizzuto, P. Pietrandrea, \& Simone (Eds.), Verbal and Signed languages: Comparing Structure, Constructs and Methodologies (pp. 13-33.). Berlin: de Gruyter.

de Leeuw, J. R. (2015). jsPsych: A JavaScript library for creating behavioral experiments in a Web browser. Behavior Research Methods, 47(1), 1-12. https://doi.org/10.3758/s13428-0140458-y

Dryer, M. (2005). Order of subject, object, and verb. In The world atlas of language structures (pp. 330-333).

Fischer, S. (1975). Influences on word order change in American Sign Language. In C. Li (Ed.), Word Order and Word Order Change (pp. 3-25). Austin, TX: University of Texas Press.

Futrell, R., Hickey, T., Lee, A., Lim, E., Luchkina, E., \& Gibson, E. (2015). Cross-linguistic gestures reflect typological universals: A subject-initial, verb-final bias in speakers of diverse languages. Cognition, 136, 215-221. https://doi.org/10.1016/j.cognition.2014.11.022

Gell-Mann, M., \& Ruhlen, M. (2011). The origin and evolution of word order. Proceedings of the National Academy of Sciences, 108(42), 17290-17295. 
PsyArxiv preprint first posted online March 3, 2017, http://osf.io/vaqk3/. The copyright holders for this preprint (which was not peer-reviewed) are the authors. It is made available under a CC-BY-NC-ND 4.0 International license.

https://doi.org/10.1073/pnas.1113716108

Gelman, R., \& Butterworth, B. (2005). Number and language: How are they related? Trends in Cognitive Sciences, 9(1), 6-10.

Gibson, E., Bergen, L., \& Piantadosi, S. T. (2013). Rational integration of noisy evidence and prior semantic expectations in sentence interpretation. Proceedings of the National Academy of Sciences, 110(20), 8051-8056. https://doi.org/10.1073/pnas.1216438110

Gibson, E., Piantadosi, S. T., Brink, K., Bergen, L., Lim, E., \& Saxe, R. (2013). A NoisyChannel Account of Crosslinguistic Word-Order Variation. Psychological Science, 24(7), 1079_ 1088. https://doi.org/10.1177/0956797612463705

Givón, T. (1979). On understanding grammar. New York, NY: Academic Press.

Goldin-Meadow, S., \& Feldman, H. (1977). The development of language-like communication without a language model. Science, 197, 401-403.

Goldin-Meadow, S., So, W. C., Özyürek, A., \& Mylander, C. (2008). The natural order of events: How speakers of different languages represent events nonverbally. Proceedings of the National Academy of Sciences, 105(27), 9163.

Gureckis, T. M., Martin, J., McDonnell, J., Rich, A. S., Markant, D., Coenen, A., ... Chan, P. (2015). psiTurk: An open-source framework for conducting replicable behavioral experiments online. Behavior Research Methods, 1-14. https://doi.org/10.3758/s13428-015-0642-8

Hall, M. L., Ferreira, V. S., \& Mayberry, R. I. (2014). Investigating Constituent Order Change With Elicited Pantomime: A Functional Account of SVO Emergence. Cognitive Science, n/an/a. https://doi.org/10.1111/cogs.12105 
PsyArxiv preprint first posted online March 3, 2017, http://osf.io/vaqk3/. The copyright holders for this preprint (which was not peer-reviewed) are the authors. It is made available under a CC-BY-NC-ND 4.0 International license.

Hall, M. L., Mayberry, R. I., \& Ferreira, V. S. (2013). Cognitive constraints on constituent order:

Evidence from elicited pantomime. Cognition, 129(1), 1-17.

https://doi.org/10.1016/j.cognition.2013.05.004

Jaeger, T. F. (2010). Redundancy and reduction: Speakers manage syntactic information density. Cognitive Psychology, 61(1), 23-62. https://doi.org/10.1016/j.cogpsych.2010.02.002

Kocab, A., Lam, H., \& Snedeker, J. (Under Review). Cars crashing and people hugging: The effect of animacy on word order in gestural language creation.

Langus, A., \& Nespor, M. (2010). Cognitive systems struggling for word order. Cognitive Psychology, 60(4), 291-318. https://doi.org/10.1016/j.cogpsych.2010.01.004

Levy, R., Bicknell, K., Slattery, T., \& Rayner, K. (2009). Eye movement evidence that readers maintain and act on uncertainty about past linguistic input. Proceedings of the National Academy of Sciences, 106(50), 21086-21090. https://doi.org/10.1073/pnas.0907664106

Lillo-Martin, D., \& Klima, E. S. (1990). Pointing out differences: ASL pronouns in syntactic theory. In Theoretical Issues in Sign Language Research, Volume 1: Linguistics (pp. 191-210). Chicago: University of Chicago Press.

Mahowald, K., Fedorenko, E., Piantadosi, S. T., \& Gibson, E. (2013). Info/information theory: Speakers choose shorter words in predictive contexts. Cognition, 126(2), 313-318. https://doi.org/10.1016/j.cognition.2012.09.010

McKee, R., \& Napier, J. (2002). Interpreting into International Sign Pidgin: An analysis. Sign Language \& Linguistics, 5(1), 27-54. https://doi.org/10.1075/s1l.5.1.04mck

Meir, I., Aronoff, M., Börstell, C., Hwang, S.-O., Ilkbasaran, D., Kastner, I., ... Sandler, W. 
PsyArxiv preprint first posted online March 3, 2017, http://osf.io/vaqk3/. The copyright holders for this preprint (which was not peer-reviewed) are the authors. It is made available under a CC-BY-NC-ND 4.0 International license.

(2017). The effect of being human and the basis of grammatical word order: Insights from novel communication systems and young sign languages. Cognition, 158, 189-207. https://doi.org/10.1016/j.cognition.2016.10.011

Meir, I., Lifshitz, A., Ilkbasaran, D., \& Padden, C. A. (2010). The interaction of animacy and word order in human languages: A study of strategies in a novel communication task. Presented at the 8th international conference on the evolution of language, Utrecht, Germany.

Napoli, D. J., \& Sutton-Spence, R. (2014). Order of the major constituents in sign languages: implications for all language. Frontiers in Psychology, 5. https://doi.org/10.3389/fpsyg.2014.00376

Newmeyer, F. (2000). On the reconstruction of "proto-world" word order. In C. Knight, M. Studdert-Kennedy, \& J. Hurford (Eds.), The Evolutionary Emergence of Language: Social Function and the Origins of Liguistic Form (pp. 372-388). Cambridge: Cambridge University Press.

Piantadosi, S. T., Tily, H., \& Gibson, E. (2011). Word lengths are optimized for efficient communication. Proceedings of the National Academy of Sciences, 108(9), 3526-3529. https://doi.org/10.1073/pnas.1012551108

Piantadosi, S. T., Tily, H., \& Gibson, E. (2012). The communicative function of ambiguity in language. Cognition, 122(3), 280-291. https://doi.org/10.1016/j.cognition.2011.10.004

Schouwstra, M., \& de Swart, H. (2014). The semantic origins of word order. Cognition, 131(3), 431-436. https://doi.org/10.1016/j.cognition.2014.03.004

Shannon, C. E. (1949). Communication in the Presence of Noise. Proceedings of the IRE, 37(1), 
PsyArxiv preprint first posted online March 3, 2017, http://osf.io/vaqk3/. The copyright holders for this preprint (which was not peer-reviewed) are the authors. It is made available under a CC-BY-NC-ND 4.0 International license.

10-21. https://doi.org/10.1109/JRPROC.1949.232969

Taub, S. (2001). Language from the Body: Iconicity and Metaphor in American Sign Language. Cambridge: Cambridge University Press. 